\title{
Article \\ Adaptive Cruise Control with Look-Ahead Anticipation for Driving on Freeways
}

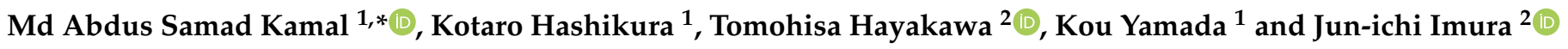 \\ 1 Graduate School of Science and Technology, Gunma University, Kiryu 376-8515, Japan; \\ k-hashikura@gunma-u.ac.jp (K.H.); yamada@gunma-u.ac.jp (K.Y.) \\ 2 School of Engineering, Tokyo Institute of Technology, Tokyo 152-8552, Japan; \\ hayakawa@sc.e.titech.ac.jp (T.H.); imura@sc.e.titech.ac.jp (J.-i.I.) \\ * Correspondence: maskamal@gunma-u.ac.jp
}

Citation: Kamal. M.A.S.; Hashikura, K.; Hayakawa, T.; Yamada, K.; Imura, J.-i. Adaptive Cruise Control with Look-Ahead Anticipation for Driving on Freeways. Appl. Sci. 2022, 12, 929. https://doi.org/10.3390/ app12020929

Academic Editor: Emanuele Carpanzano

Received: 17 December 2021

Accepted: 14 January 2022

Published: 17 January 2022

Publisher's Note: MDPI stays neutral with regard to jurisdictional claims in published maps and institutional affiliations.

Copyright: (c) 2022 by the authors. Licensee MDPI, Basel, Switzerland. This article is an open access article distributed under the terms and conditions of the Creative Commons Attribution (CC BY) license (https:// creativecommons.org/licenses/by/ $4.0 /)$.

\begin{abstract}
This paper presents Adaptive Cruise Control (ACC) with look-ahead anticipation, based on the model of ACC used in recent commercial vehicles, to take early decisions in driving a vehicle on the freeway. The existing ACC found in the high-end cars has limited operating range as it often fails to respond smoothly in advance behind a decelerating vehicle. Although advanced techniques, such as model predictive control (MPC), can significantly improve a vehicle's driving performance, they are associated with high computational complexity and have limited scopes for practical implementation. The proposed look-ahead anticipatory scheme of ACC predicts the relative states of the preceding vehicle using a conditional persistence prediction technique in an adaptive short horizon. With negligible computation cost, it determines the control input using parametric functions prudently for improving driving performance. The proposed scheme is evaluated on multiple vehicles in typical traffic scenarios to examine individual driving behavior and the stability of a vehicle string. Finally, we investigate the influences of a small part of vehicles with the proposed ACC on overall traffic using the AIMSUN traffic simulator and compare performances of overall traffic.
\end{abstract}

Keywords: look-ahead anticipatory driving; adaptive cruise control (ACC); automated driving; predictive control

\section{Introduction}

Basic human car-following behavior and its impact on traffic stability have been studied extensively in various contexts [1-4]. Automated driving technologies, mostly aiming at safe maneuver and rider comforts, have received increasing attention in recent years. Automation can partly alleviate the stress of a driver who requires a high level of concentration for driving explicitly in dense traffic. One of the key functionalities of any driving automation system is to regulate the gap with the preceding vehicle. Adaptive cruise control (ACC), which is such a commercially available automation technology equipped in most existing high-end cars, can dynamically control the vehicle speed and maintain a safe gap. Vehicles with ACC can provide smoother driving with less acceleration variation that directly enhances driving comforts and environmental benefits [5]. ACC has the potential to improve driving behavior, ameliorate fuel consumption, and provide driver comfort through in-vehicle driver assistance [6].

The principal objective of ACC is to offer driver assistance by adjusting both the throttle and brake of a vehicle within the restricted acceleration range in a high-speed (e.g., above $40 \mathrm{~km} / \mathrm{h}$ ) driving environment. Recent research on ACC is more focused on the extended range of driving (e.g., stop and go traffic) $[7,8]$ and enhancing the efficiency of traffic flows [9-11]. By setting the parameters of ACC, e.g., time gap, it is possible to improve traffic flow capacity and performance. In particular, using bi-directional sensing with inter-vehicle communication, cooperative ACC, called CACC, can control vehicles in a platoon by keeping a short time gap between the pairs of vehicles and improving 
traffic capacity significantly [9,12-15]. CACC can be implemented only when a group of dedicated vehicles with connectivity closely move on the same lane and share the same route without the interference or mixing of traditional vehicles. The prospects of CACC are further limited in heterogeneous traffic as the formation of a platoon or resizing a running platoon by incorporating diverse types of vehicles is technically not feasible. For these reasons, CACC has never been implemented on commercial cars, except for some limited experimental purposes [16-18]. Recent research on ACC or car-following schemes using the connectivity of automated vehicles received tremendous interest. A major part of such research introduces connectivity features to develop an enhanced car-following scheme using modified human car-following models [19-21]. A robust car-following control strategy is presented considering uncertainty and communication delay [4]. Although research on such connectivity-based driving is necessary for future transportation systems, research related to existing automated car-following technology or ACC found on commercial cars is very limited $[22,23]$.

Basic ACC studies focus on different spacing or time gap policies for individual vehicles without cooperating or communicating with the other vehicles. Several works examined the impacts of ACC-equipped vehicles (or simply, ACC vehicles) with adaptive time gaps on traffic flow at their various penetration rates $[9,11,12,24]$. The operating ranges of some commercial ACC are limited to moderate to high speed, e.g., above $40 \mathrm{~km} / \mathrm{h}$. ACC often needs to be deactivated when the preceding vehicle brakes sharply unless a very conservative spacing is used [5,22]. Furthermore, vehicles with such ACC are not always energy efficient, and they may cause shock waves or congestion due to the presence of disturbances in dense traffic. The offline multi-objective optimization technique was used to optimize parameters in order to have a suitable trade-off between safety and performance objectives [25]. These ACCs are unable to finetune their driving behavior dynamically by considering the changing trends of the traffic ahead; therefore, their performances are affected greatly in transient conditions.

Various advanced automated vehicle control systems, e.g., MPC, have been investigated recently to overcome the limitations of traditional driving or ACC [10,26-29]. Comfortable and efficient driving behaviors can be realized by anticipating the traffic ahead using information via inter-vehicle communication and taking appropriate control action to drive a vehicle smoothly and improve traffic flows [30,31]. In these MPC-based driving systems, using the predicted state of the preceding vehicle, an optimization problem is solved to obtain the control input of the vehicle. Hence, the solution provides anticipatory car-following behavior that improves the driving efficiency of a vehicle by dynamically tuning spacing and speed.

In contrast to these advanced vehicle control, traditional ACC decides the vehicle control input according to the current state of the preceding vehicle; therefore, the vehicle cannot avoid aggressive braking often in varying traffic flow conditions $[10,22]$. Due to high computational cost and complexity in obtaining reliable solutions, MPC is not easy to implement on vehicles despite a potential comfort gain for the driver and environmental benefits. Therefore, the use of these advanced driving systems remains limited mostly in theoretical and simulation studies. Despite being a decades-old technology, none of the existing ACC found on commercial cars uses the advanced optimization-based decision technique. A very recent experimental study on seven different 2018-model-year vehicles with ACC reveals that none of these vehicles are even string stable, i.e., the ACCs can operate the vehicles only at steady speed above some high level and need to be deactivated in complex maneuvers [32].

Pointing out the potentials and limitations of the predictive optimal control schemes, a simple look-ahead car-following scheme using the intelligent driver model (IDM) [33] was proposed that takes control decision by predicting the behavior of the preceding vehicle [34]. A detailed investigation has been conducted recently by applying such a look-ahead car following with the existing driving schemes to observe their impacts on the fuel consumption, the overall traffic flow, and utilization of the intersection at various 
penetration rates [35]. In the context of signalized intersection and low-speed driving on the urban roads, the model of commercial ACC with such a look-ahead scheme does not perform well due to its frequent and long deactivation periods. Therefore, how anticipatory driving features influence the operating scope and the performance of existing ACC on the freeway with designed operating speed have not been investigated yet.

This paper presents a look-ahead scheme of ACC by extending the decision process of an existing ACC available on commercial high-end cars. In the context of freeway driving, the future position and speed of the preceding vehicle after a short period are predicted using a conditional persistence prediction technique and then used to decide the control input of the vehicle by using the model of a commercial ACC that was calibrated using experimental driving data [22]. Although the look-ahead feature of the proposed ACC is inspired by the MPC-based driving scheme, it completely differs from MPC in the sense that the driving decision is taken in real time using parametric functions instead of rigorous numerical optimization. Therefore, the main contribution is the look-ahead framework that connects prediction and decision-making procedures to the existing ACC employing parametric functions (i.e., without involving numerical optimization) for realtime computation to provide a partly similar behavior pattern of the MPC schemes to some extent. A time-varying prediction horizon and the conditional persistent prediction technique were introduced to keep the look-ahead predicted states reliably accurate despite varying traffic conditions.

The proposed ACC is implemented in a moving horizon fashion, where the control decision is taken considering a look-ahead horizon, but the process is repeated at a smalltime step that is a fraction of the horizon. Such feedback features enable the ACC to adapt to the actual traffic conditions timely. Furthermore, this look-ahead technique can be used with any ACC reviewed above to enhance their ability to cope with the varying traffic conditions by taking early anticipatory action. At first, the ability of the proposed ACC in improving driving behavior is observed by measuring a typical driving performance index that is usually used in an optimal driving system. Next, it is evaluated on a group of vehicles in changing situations of dense traffic, where the preceding vehicle accelerates or brakes at different magnitudes. Finally, using an experimental setup in AIMSUN microscopic traffic simulator, the influences of a small percentage of vehicles with the proposed ACC on the overall traffic are evaluated on a freeway. From these evaluations, the potential benefits of the proposed ACC over the original ACC have been investigated.

The structure of this paper is as follows. The next section briefly reviews the vehicle dynamics and traditional ACC and describes the proposed look-ahead scheme of ACC. Section 4 includes the evaluation settings and describes the evaluation results in three different test scenarios. Finally, Section 4 concludes the contributions of this paper.

\section{Look-Ahead Anticipatory Scheme of Adaptive Cruise Control}

Consider a longitudinal motion control problem of a host vehicle. It is assumed that the lane change and lateral motion of the vehicle are controlled by its driver or an independent system. The control input is given by $a_{\mathrm{h}}$ (suffix $\mathrm{h}$ refers to the host vehicle), and the state vector $z_{\mathrm{h}}=\left[x_{\mathrm{h}}, v_{\mathrm{h}}\right]^{\top} \in \mathbb{R}^{2}$ consists of position $x_{\mathrm{h}}$ and speed $v_{\mathrm{h}}$ of the host vehicle. The state of the control problem is given as follows.

$$
\dot{z}_{\mathrm{h}}(t)=\left[v_{\mathrm{h}}(t), a_{\mathrm{h}}(t)\right]^{\top} .
$$

The control input $a_{\mathrm{h}}$ of the host vehicle needs to be decided considering the state $z_{\mathrm{p}}=\left[x_{\mathrm{p}}, v_{\mathrm{p}}\right]^{\top} \in \mathbb{R}^{2}$ of its preceding vehicle (denoted by suffix $\mathrm{p}$ ). A human car following model or ACC can be employed to determine control input $a_{\mathrm{h}}$ to drive the host vehicle. For real implementation of ACC, a low-level controller should apply appropriate torque commands (i.e., considering the dynamics including delay and inertia of the vehicles) to the actuators. 


\subsection{Review of an Existing ACC}

The model of a commercially used ACC system is reviewed briefly according to [22,23] for the convenience in developing its look-ahead scheme. The model is developed based on experimental data taken from the ACC equipped vehicles on test runs [22]. Acceleration of the host vehicle with ACC for regulating the gap or cruising was modeled as a function of the state (i.e., position and speed) of the host vehicle and the state of its preceding vehicle, if it exists within a specified range of $R_{\text {th }}$. Acceleration decided by ACC at time $t$ can generally be provided by a function $f_{\text {acc }}$ as follows:

$$
\begin{aligned}
a_{\mathrm{h}}(t) & =f_{\mathrm{acc}}\left(z_{\mathrm{h}}(t), z_{\mathrm{p}}(t)\right) \\
& = \begin{cases}f_{\text {free }}\left(v_{\mathrm{h}}(t)\right), & \text { for } x_{\mathrm{p}}(t)-x_{\mathrm{h}}(t)>R_{\mathrm{th}}, \\
f_{\text {regu }}\left(z_{\mathrm{h}}(t), z_{\mathrm{p}}(t)\right), & \text { otherwise, }\end{cases}
\end{aligned}
$$

where the functions $f_{\text {free }}(\cdot)$ and $f_{\text {regu }}(\cdot, \cdot)$ represent the decisions in the cruising mode and the gap-regulating mode, respectively. They are defined as follows:

$$
\begin{aligned}
f_{\text {free }}\left(v_{\mathrm{h}}\right)= & k_{0}\left(v_{\text {ref }}-v_{\mathrm{h}}\right), \\
f_{\text {regu }}\left(z_{\mathrm{h}}, z_{\mathrm{p}}\right)= & k_{1}\left(x_{\mathrm{p}}-x_{\mathrm{h}}-d_{0}+t_{\mathrm{hw}} v_{\mathrm{h}}\right) \\
& +k_{2}\left(v_{\mathrm{p}}-v_{\mathrm{h}}\right),
\end{aligned}
$$

where $d_{0}$ is a constant, $t_{\mathrm{hw}}$ is the set time headway, $k_{0}, k_{1}$, and $k_{2}$ are gains, and $v_{\text {ref }}$ is the reference speed set by the driver.

By minimizing the difference between experimental and simulated speeds using model (4) in the gap regulating mode (i.e., for $x_{\mathrm{p}}-x_{\mathrm{h}} \leq R_{\mathrm{th}}$ ), the gains are approximated as $k_{1}=0.23 \mathrm{~s}^{-2}$ and $k_{2}=0.07 \mathrm{~s}^{-1}$ [22]. For a smooth transition from the cruising mode (i.e., for $x_{\mathrm{p}}-x_{\mathrm{h}}>R_{\mathrm{th}}$ ) to the gap regulating mode by quickly reducing the speed error, a new mode called approaching mode is considered in [23]. The approaching mode starts when the gap falls within twice as large as the desired gap (i.e., $x_{\mathrm{p}}-x_{\mathrm{h}}-d_{0}<2 t_{\mathrm{hw}} v_{\mathrm{h}}$ ). When gap and speed errors simultaneously become smaller than $0.2 \mathrm{~m}$ and $0.1 \mathrm{~m} / \mathrm{s}$, respectively, the regulating mode starts. In the approaching mode, the gains of (4) are tuned as $k_{1}=0.04 \mathrm{~s}^{-2}$ and $k_{2}=0.8 \mathrm{~s}^{-1}$. Gain $k_{0}$ for the cruising mode is chosen at $0.4 \mathrm{~s}^{-1}$, which falls within the typical range found in various ACC systems [23]. The desired gap including vehicle length $d_{0}$ between vehicles at standstill is considered zero in [22] assuming that ACC is only kept active at a high speed with sufficient gaps. In order to prevent rear-end collisions with a higher clearance at low speed, $d_{0}$ was tuned in [23] as a function of vehicle speed: at speed below $10.8 \mathrm{~m} / \mathrm{s}, d_{0}=7 \mathrm{~m}$; at speed above $15 \mathrm{~m} / \mathrm{s}, d_{0}=5 \mathrm{~m}$; and at speed between $10.8 \mathrm{~m} / \mathrm{s}$ and $15 \mathrm{~m} / \mathrm{s}, d_{0}$ is linearly tuned from $7 \mathrm{~m}$ to $5 \mathrm{~m}$.

In the above-described scheme, a driver has to deactivate ACC and drive manually when the host vehicle approaches a standstill vehicle or intends to change the lane in a restricted situation. A system-initiated deactivation method is also introduced to cope with the situation when there is a high risk of rear-end collisions according to a model of the safety-critical situation. Incorporating such functionality, ACC guarantees no collision in prevailing driving conditions [23].

\subsection{Anticipatory Feature with ACC}

It has been intensively investigated that the predictive control of vehicles can provide improved driving behavior. In such predictive control schemes, using the predicted future states of the preceding vehicle, the optimal control input is obtained in such a manner that the vehicle can avoid any aggressive acceleration in subsequent time. In order to incorporate such predictive features in the existing ACC, a simple look-ahead scheme of making anticipatory driving decisions with negligible computation cost is proposed. The term look-ahead refers to making the appropriate plan by anticipating what will occur in the future. For the sake of simplicity, the proposed look-ahead anticipatory scheme of ACC is referred to as $L a-A C C$, henceforth, in the paper. In particular, as illustrated in the concept 
of the proposed La-ACC in Figure 1, the relative states of both the host and preceding vehicles are determined for a short look-ahead horizon $h_{\mathrm{la}}$, and using such a predicted state, the standard decision function of ACC is used to take a control action.

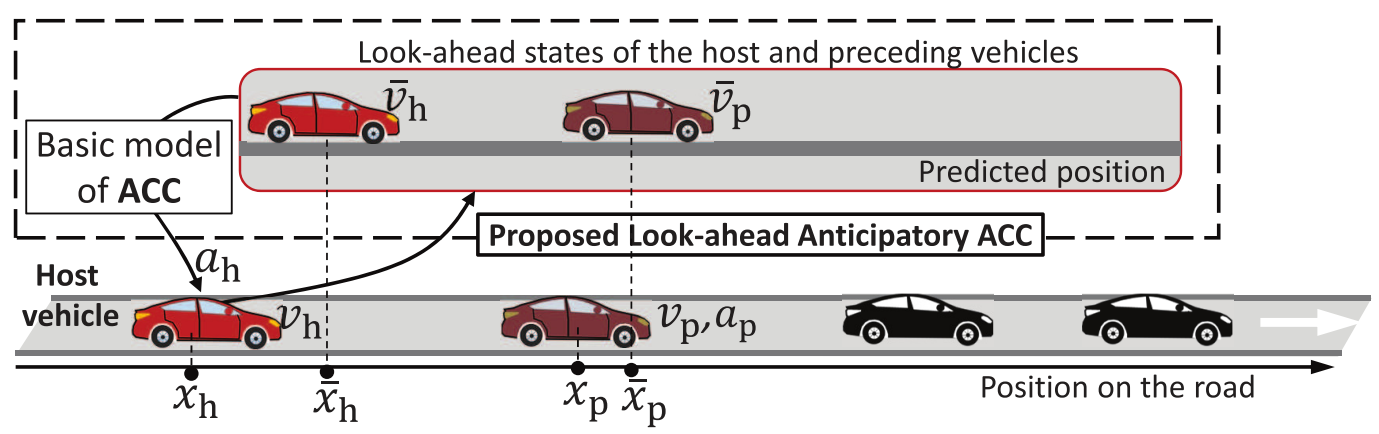

Figure 1. The decision-making process of the proposed La-ACC, where the driving decision is taken using the look-ahead or predicted states and the basic model of ACC.

Specifically, for a look-ahead horizon $h_{\mathrm{la}}(t)$, the control decision of the proposed La-ACC is described by a function $f_{\text {lacc }}$ as follows:

$$
\begin{aligned}
a_{\mathrm{h}}(t) & =f_{\text {lacc }}\left(z_{\mathrm{h}}(t), z_{\mathrm{p}}(t), \bar{a}_{\mathrm{p}}(t)\right) \\
& =f_{\mathrm{acc}}\left(\bar{z}_{\mathrm{h}}\left(t+h_{\mathrm{la}}(t)\right), \bar{z}_{\mathrm{p}}\left(t+h_{\mathrm{la}}(t)\right)\right),
\end{aligned}
$$

where $\bar{a}_{\mathrm{p}}(t)$ denotes the estimated average acceleration of the preceding vehicle in the horizon, $\bar{z}_{\mathrm{h}}(\cdot)=\left[\bar{x}_{\mathrm{h}}(\cdot), \bar{v}_{\mathrm{h}}(\cdot)\right]^{\top}$ and $\bar{z}_{\mathrm{p}}(\cdot)=\left[\bar{x}_{\mathrm{p}}(\cdot), \bar{v}_{\mathrm{p}}(\cdot)\right]^{\top}$ denote the respective lookahead states of the host and preceding vehicles. Specifically, they are obtained as follows.

$$
\begin{aligned}
& \bar{v}_{\mathrm{h}}\left(t+h_{\mathrm{la}}(t)\right)=v_{\mathrm{h}}(t), \\
& \bar{v}_{\mathrm{p}}\left(t+h_{\mathrm{la}}(t)\right)=v_{\mathrm{p}}(t)+\bar{a}_{\mathrm{p}}(t) h_{\mathrm{la}}(t), \\
& \bar{x}_{\mathrm{h}}\left(t+h_{\mathrm{la}}(t)\right)=x_{\mathrm{h}}(t)+v_{\mathrm{h}}(t) h_{\mathrm{la}}(t), \\
& \bar{x}_{\mathrm{p}}\left(t+h_{\mathrm{la}}(t)\right)=x_{\mathrm{p}}(t)+v_{\mathrm{p}}(t) h_{\mathrm{la}}(t)+0.5 \bar{a}_{\mathrm{p}}(t) h_{\mathrm{la}}^{2}(t) .
\end{aligned}
$$

La-ACC given by (5) uses the gains and parameters of the original ACC but with the predicted relative states of the host and the preceding vehicles. At low speeds with a short gap, a long look-ahead horizon may cause undesired oscillations, since the acceleration of the preceding vehicle is often uncertain and noisy. Therefore, the look-ahead horizon $h_{\text {la }}$ is tuned dynamically as follows:

$$
h_{\mathrm{la}}(t)= \begin{cases}h_{\max } \beta^{-1} v_{\mathrm{h}}(t), & \text { if } v_{\mathrm{h}}(t) \leq \beta, \\ h_{\max }, & \text { otherwise }\end{cases}
$$

where $\beta$ is a constant, and $h_{\max }$ is the maximum look-ahead horizon in second. Depending on speed $v_{\mathrm{h}}$, look-ahead horizon $h_{\mathrm{la}}$ is tuned linearly from 0 to $h_{\mathrm{max}}$ when the speed varies from 0 to $\beta$. Such tuning at a low speed helps avoid any oscillatory decision due to the presence of the preceding vehicle at a short gap.

The key parameter of this look-ahead approach is the acceleration of the preceding vehicle $\bar{a}_{\mathrm{p}}(t)$. In the case of connected-cooperative driving, such information can be provided directly by the preceding vehicle. In the traditional unconnected driving scenario considered in this study, $\bar{a}_{\mathrm{p}}(t)$ is estimated by using the recent speeds of the preceding vehicle according to a conditional persistence prediction technique [30]. Instead of capturing any momentary speed fluctuations, we consider a consistently accelerating condition over the last $2 \tau \mathrm{s}$ and propose estimating $\bar{a}_{\mathrm{p}}(t)$ as follows:

$$
\bar{a}_{\mathrm{p}}(t)=\hat{a}_{\mathrm{p}}(t) \theta_{1}\left(v_{\mathrm{p}}(t)\right) \mathrm{e}^{-\alpha\left(\tau+h_{\mathrm{la}} / 2\right)}
$$


where $\theta_{1}(\cdot)$ is a limiting function; using the natural exponent e, constant $\alpha$ defines the decaying factor at the mid of look-ahead horizon $h_{\mathrm{la}} / 2$, and the unconditional estimated acceleration $\hat{a}_{\mathrm{p}}(t)$ at $t$ with the limiting function $\theta_{2}(\cdot)$ is provided by the following.

$$
\hat{a}_{\mathrm{p}}(t)=a_{\mathrm{p}}(t-\tau)+\theta_{2} \dot{a}_{\mathrm{p}}(t-\tau) .
$$

In (12), the recent acceleration $a_{\mathrm{p}}(\cdot)$ and its rate $\dot{a}_{\mathrm{p}}(\cdot)$ at $t-\tau$ is obtained as follows.

$$
\begin{aligned}
& a_{\mathrm{p}}(t-\tau)=\left(v_{\mathrm{p}}(t)-v_{\mathrm{p}}(t-\tau)\right) / \tau, \\
& \dot{a}_{\mathrm{p}}(t-\tau)=\left(v_{\mathrm{p}}(t)-2 v_{\mathrm{p}}(t-\tau)+v_{\mathrm{p}}(t-2 \tau)\right) / 2 \tau .
\end{aligned}
$$

The limiting functions $\theta_{1}(\cdot), \theta_{2}(\cdot)$ that impose the condition on persistence prediction are defined as follows.

$$
\begin{aligned}
& \theta_{1}(\phi)= \begin{cases}1, & \text { for } 0<\phi<V_{\max } \\
0, & \text { otherwise }\end{cases} \\
& \theta_{2}(\phi)= \begin{cases}-\delta_{\max }, & \text { for } \phi<-\delta_{\max } \\
\delta_{\max ,} & \text { for } \phi>\delta_{\max } \\
\phi, & \text { otherwise. }\end{cases}
\end{aligned}
$$

where constant $V_{\max }$ denotes the limit of speed, and $\delta_{\max }$ denotes the limit of the acceleration rate. The above-described conditional persistence prediction technique uses only a few parameters with very specific meaning and, in typical driving conditions, can closely predict a vehicle's state for a short look-ahead horizon as verified on experimental driving data [30].

The proposed La-ACC does not guarantee collision avoidance in transient conditions since most parameters are the same as the original ACC. Therefore, sometimes it needs to be deactivated in some critical situations, e.g., at low speed, lane change, and shock-wave. It is assumed that the driver can take control of the vehicle immediately in such a case. Once the vehicle returns to a steady condition, La-ACC can be reactivated again.

\section{Evaluation of the Proposed La-ACC}

By using microscopic traffic simulation, the proposed La-ACC is evaluated, and its performances for controlling multiple vehicles in typical freeway congested traffic are compared with the existing ACC as described in Section 2.1. Most parameters are kept the same as that of the original ACC, and they are directly taken from [22,23]. In particular, maximum acceleration and braking are set at $2 \mathrm{~m} / \mathrm{s}^{2}$ and $-4 \mathrm{~m} / \mathrm{s}^{2}$, respectively. The parameters $\beta$ and $h_{\text {max }}$ are determined by applying sensitivity analysis by balancing both performance enhancement and oscillation suppression in transient situations. A long look-ahead horizon (more than the usual time gap of a driver or driving system) may cause speed oscillations of the vehicle in transient traffic conditions. In particular, the typical time gap of ACC, which can be as low as $1 \mathrm{~s}$, and the look-ahead parameters of (10) are set at $\beta=4 \mathrm{~m} / \mathrm{s}$ and $h_{\max }=1$. The parameters of the conditional persistent prediction are tuned as $\tau=1 \mathrm{~s}, \alpha=0.45$, and $\delta=2 \mathrm{~m} / \mathrm{s}^{2}$, which ensure a mean square speed prediction error of $0.26 \mathrm{~km} / \mathrm{h}$ for the maximum look-ahead horizon of $h_{\max }=1 \mathrm{~s}$ based on observation on the experimental driving data of about $8 \mathrm{~km}$ of driving [36]. The maximum speed $V_{\max }$ is set at the maximum speed limit of the roads. The built-in fuel consumption model in AIMSUN with calibrated parameters based on the Ford Fiesta car, a light-duty vehicle with an internal combustion engine, is used for evaluation in this study [37].

It is assumed that ACC is deactivated by the driver when the required braking rate exceeds $50 \%$ of the maximum braking limits due to the presence of a slow or braking preceding vehicle; subsequently, the human driver takes control of the vehicle. In particular, in this study, we considered IDM+ (IDM plus, a modified version of IDM) [38] as the human driver model, which provides better flowing behavior of vehicles in the synchronized 
traffic, as it was used with ACC in [23]. When the vehicle returns at a steady condition (with acceleration less than $0.2 \mathrm{~m} / \mathrm{s}^{2}$ ), ACC is reactivated after waiting for $30 \mathrm{~s}$.

\subsection{Evaluation of the Proposed La-ACC on a Single Vehicle}

At first, the influence of the maximum look-ahead horizon $h_{\max }$ on the generated acceleration by La-ACC is observed and shown in Figure 2. In this observation, the host vehicle is running at a speed of $80 \mathrm{~km} / \mathrm{h}$ with a gap of $30 \mathrm{~m}$ from its preceding vehicle. Three different relative speeds and, for each, three distinct accelerating cases of the preceding vehicle are separately analyzed. Figure 2 a shows the case when both the preceding and host vehicles are running at the same speed of $80 \mathrm{~km} / \mathrm{h}$ (i.e., $\Delta V_{\mathrm{p}}=v_{\mathrm{h}}-v_{\mathrm{p}}=0$ ). For instant acceleration $a_{\mathrm{p}}=0$ of the preceding vehicle, i.e., both vehicles are at equilibrium states, La-ACC generates zero acceleration for any look-ahead horizon (i.e., for 0 to 2 sec as shown in the horizontal axis). Note that when the look-ahead horizon $h_{\mathrm{la}}$ is zero, La-ACC is exactly equivalent to ACC. However, when $a_{\mathrm{p}}=-0.25$ or $a_{\mathrm{p}}=0.25$ for the same speed of both vehicles, La-ACC with a longer horizon generates acceleration by aligning to the decelerating/accelerating trend of the preceding vehicle.

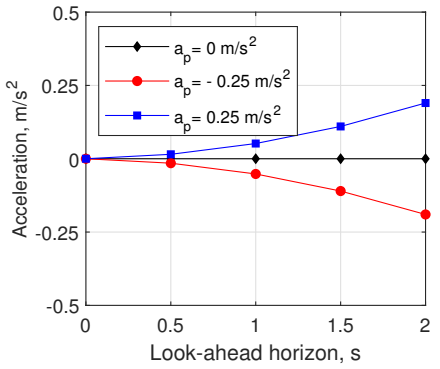

(a) $\Delta \mathrm{V}_{\mathrm{p}}=0 \mathrm{~km} / \mathrm{h}$

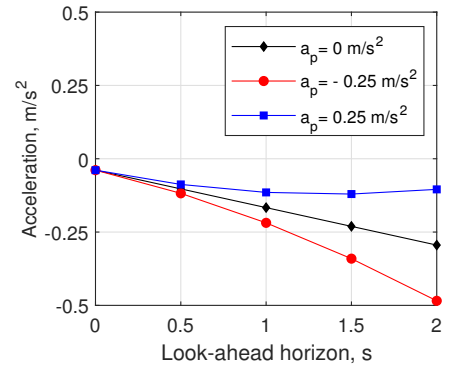

(b) $\Delta V_{p}=-2 \mathrm{~km} / \mathrm{h}$

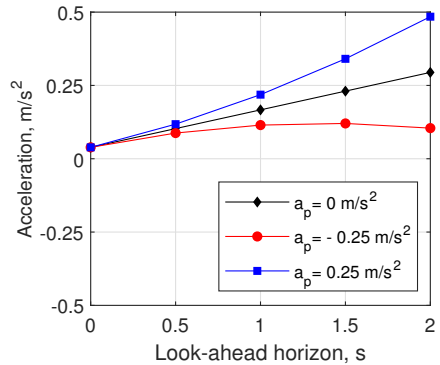

(c) $\Delta \mathrm{V}_{\mathrm{p}}=+2 \mathrm{~km} / \mathrm{h}$

Figure 2. Acceleration characteristics of La-ACC for varying look-ahead horizon at different speeds and accelerating conditions of the preceding vehicle. The object vehicle is at $80 \mathrm{~km} / \mathrm{h}$ speed with a gap of $30 \mathrm{~m}$ from the preceding vehicle.

Figure $2 b, c$ show similar cases when the preceding vehicle has a relative speed $\Delta V_{\mathrm{p}}=-2 \mathrm{~km} / \mathrm{h}$ and $\Delta V_{\mathrm{p}}=2 \mathrm{~km} / \mathrm{h}$, respectively. Even when $a_{\mathrm{p}}=0$, La-ACC exhibits higher sensitivity than ACC by reflecting the look-ahead states. Often, the speed of a vehicle does not change monotonically in varying dense traffic; therefore, a long horizon may not be suitable for realizing smooth driving.

Next, La-ACC with $1 \mathrm{~s}$ look-ahead horizon is evaluated by controlling a host vehicle in two typical scenarios, as shown in Figure 3. In addition, by visualizing the speed and acceleration characteristics of the host vehicle, the quality of driving is also evaluated quantitatively by measuring its performance index and fuel consumption. A typical performance index $J\left(v_{\mathrm{h}}(t), a_{\mathrm{h}}(t)\right)$, generally employed in the optimal vehicle control problem, is defined as the integral of the weighted driving cost as follows:

$$
J(v(t), a(t))=\int_{t=0}^{30}\left(w\left(V_{\max }-v_{\mathrm{h}}(t)\right)^{2}+a_{\mathrm{h}}^{2}(t)\right) \mathrm{d} t
$$

for this evaluation of $30 \mathrm{~s}$ of driving. The parameters are set as $w=0.001$ and $V_{\max }=27.78 \mathrm{~m} / \mathrm{s}$ (same as the maximum speed of the preceding vehicle). A low value of $J$ is desired as it indicates less deviation from the desired speed and low acceleration or braking. Although $J$ does not include the vehicle's fuel consumption, the inclusion of the square of speed deviation and the square of acceleration relates $J$ with fuel consumption indirectly as found from various fuel consumption models, e.g., [37,39]. In Figure 3, Scenario I shows the case when the preceding vehicle speeds up from $65 \mathrm{~km} / \mathrm{h}$ to $100 \mathrm{~km} / \mathrm{h}$ in 0 to $15 \mathrm{~s}$, and Scenario II shows the case when the preceding vehicle slows down from $100 \mathrm{~km} / \mathrm{h}$ to $80 \mathrm{~km} / \mathrm{h}$ in 0 to $8 \mathrm{~s}$. The instantaneous speed and acceleration characteristics of La-ACC and ACC, taken from independent simulation for the same condition, are shown by red and blue curves, 
respectively, in each scenario. The speed of the La-ACC vehicle results in less overshoot and converges earlier than the ACC vehicle. For the observed period of $30 \mathrm{~s}, \mathrm{La}-\mathrm{ACC}$ attains a better performance index and lower fuel consumption compared to ACC. Both qualitative and quantitative comparisons indicate the advantages of La-ACC over ACC.

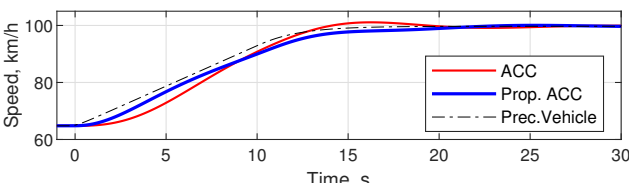

(a)

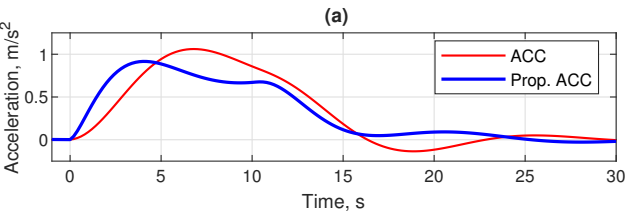

(b)

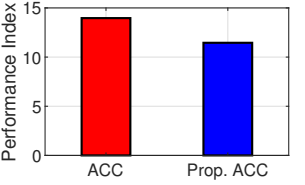

(c)

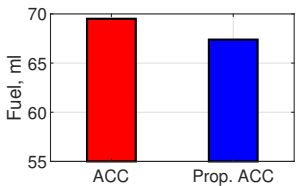

Scenario I

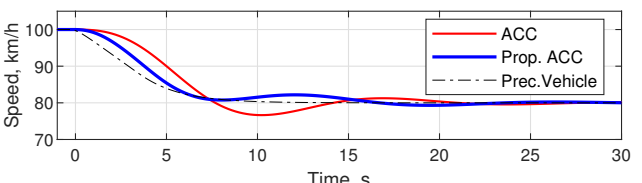

(a)

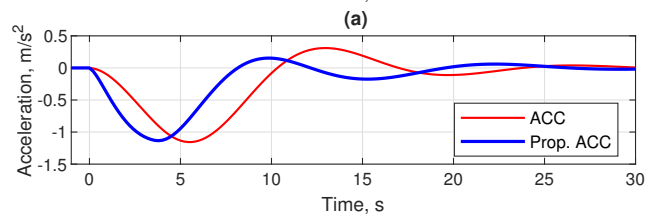

(b)

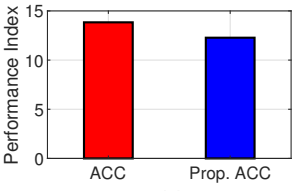

(c) (d)

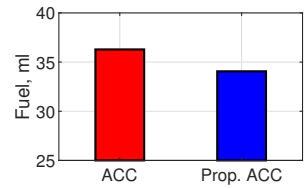

Scenario II

Figure 3. Evaluation of La-ACC and ACC in a simple car-following task in two scenarios. (a) Speed (speed of the preceding vehicle is shown by the dotted curve), (b) acceleration, (c) performance index, and (d) fuel consumption.

\subsection{Evaluation of La-ACC on Multiple Vehicles}

A vehicle usually influences the behavior of its followers and sometimes may trigger shockwaves in the following traffic that may eventually turn into a breakdown. Therefore, it is crucial to investigate the relative performances of La-ACC and examine the stability of the vehicles in dense traffic with varying speeds. We have conducted such a study and for a fair evaluation compared the performance with the recent ACC described above. Figure $4 \mathrm{a}, \mathrm{b}$ illustrate the driving characteristics of a platoon of ten vehicles using ACC and La-ACC, respectively. In each case, the lead (first) vehicle slowly increases its speed from $80 \mathrm{~km} / \mathrm{h}$ to $100 \mathrm{~km} / \mathrm{h}$ in $10 \mathrm{~s}$ and later slows down to $70 \mathrm{~km} / \mathrm{h}$ in $30 \mathrm{~s}$. Both acceleration and deceleration are smooth with very low magnitudes (within $\pm 0.4 \mathrm{~m} / \mathrm{s}^{2}$ ). The relative position (the distance from the first vehicle), speed, and acceleration of these vehicles are compared. As in Figure 4a, the ACC vehicles follow the respective preceding vehicles by causing speed oscillations, which propagate from the second to tenth vehicles. Despite the moderate acceleration of the first vehicle, the eighth to tenth vehicles have to brake aggressively by deactivating ACC temporarily as marked by the red color. These results accord with the results of [22], where they tested a platoon of five vehicles using ACC and found similar propagation of speed oscillations.

In contrast, the La-ACC vehicles have cautiously followed their respective preceding vehicles and could keep their acceleration limited, as shown in Figure $4 \mathrm{~b}$. For this low magnitude of braking by the first vehicle, the La-ACC vehicles are found fully string table as the magnitudes of their acceleration are never amplified in the following vehicles. Consequently, La-ACC remains activated in the entire driving period, i.e., La-ACC has enhanced the automated driving operating range compared with ACC for the same limits of acceleration and braking. 


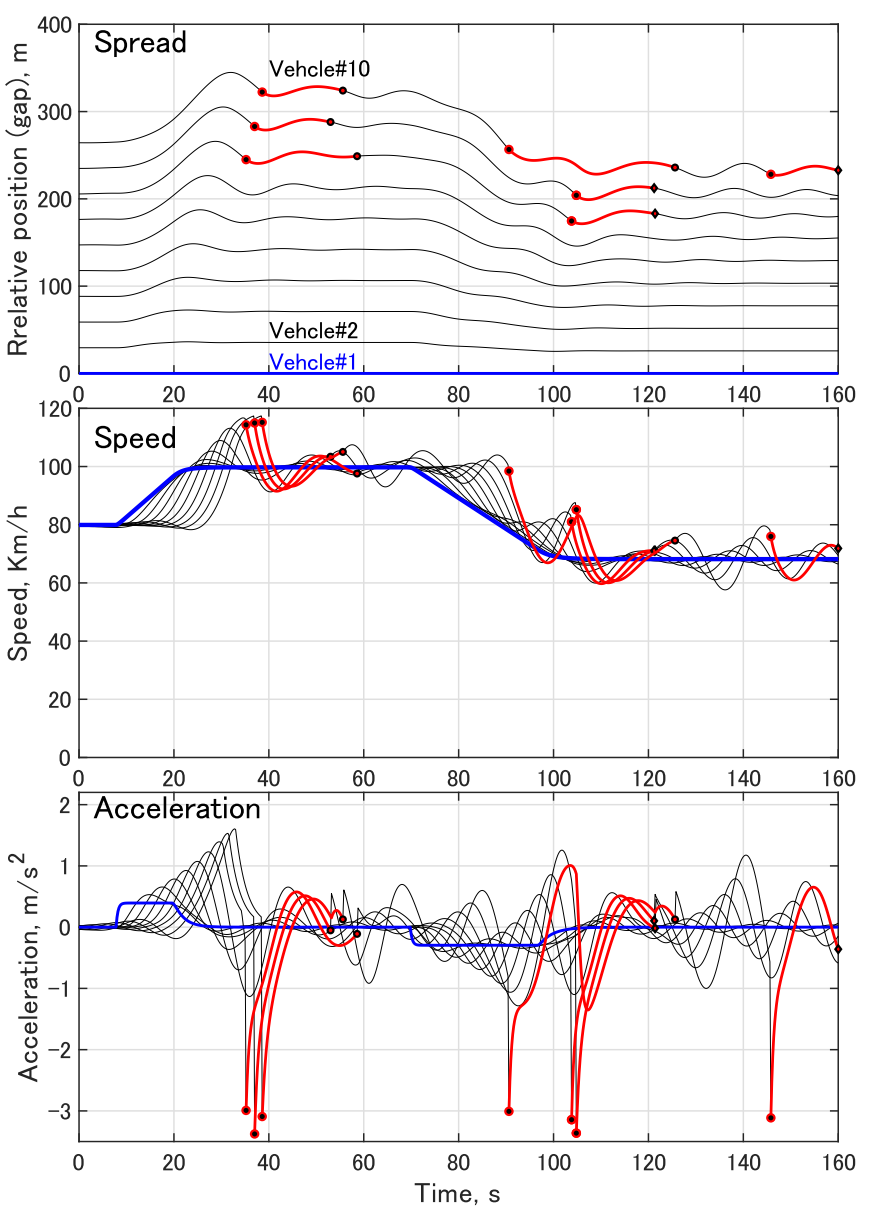

(a) ACC
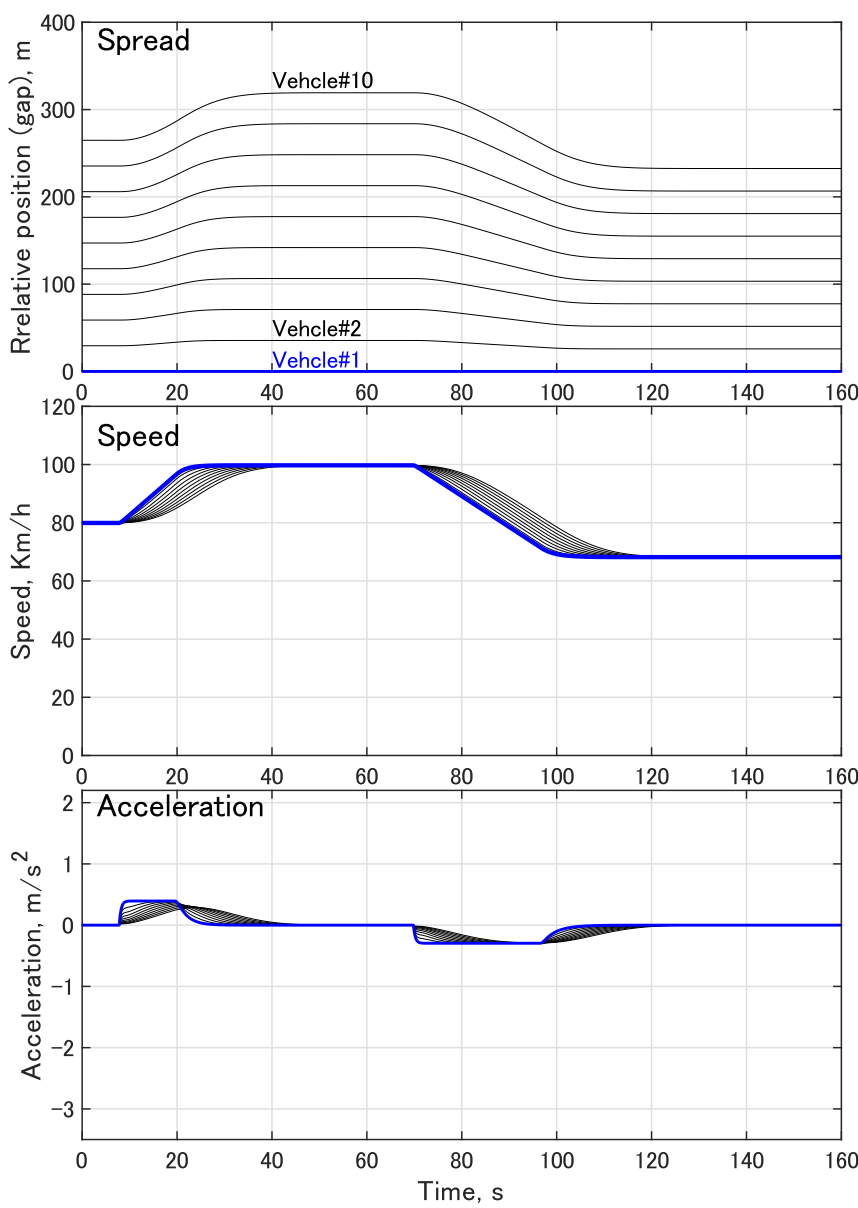

(b) Proposed La-ACC

Figure 4. Flowing behavior of ten vehicles in a platoon, where they are all controlled by (a) ACC and (b) La-ACC. The red curves show the deactivated period of ACC when they are driven by a human driver.

A similar observation with different levels of acceleration and braking by the preceding vehicle is given in Appendix A. From these observations, it is clear that our approach ensures better stability of the vehicles, i.e., without a need for deactivation when the leading vehicle changes its speed moderately, resulting in smoother flows of the vehicles with less fuel consumption than the ACC vehicles. However, when the leading vehicle brakes extensively, the La-ACC has to be deactivated similarly to ACC. Therefore, it can be concluded that our proposed approach yields almost the same or better performances compared to the recent ACC in terms of average speed, fuel consumption, and stable operating range.

\subsection{Impact of La-ACC Vehicles on Freeway Traffic}

Although numerical simulation conducted in controlled scenarios using a few vehicles, as described above, is a typical evaluation approach found in the literature, we consider further evaluation in more realistic traffic using a traffic simulator. Note that experimenting with any driving system by applying on many cars in traffic is not feasible at this point in time.

In real traffic, a vehicle often necessitates braking aggressively, e.g., for cut-in, merging, or any emergency. In such situations, La-ACC may be required to be deactivated too. Furthermore, the estimated acceleration of the preceding vehicle, hence the corresponding look-ahead state, deviates from the actual value found later. Such deviations may affect the performance of the La-ACC vehicle. Considering these facts, La-ACC vehicles' behavior is 
investigated in naturalistic traffic using the AIMSUN Microscopic Traffic Simulator [40]. In the simulator, arbitrary vehicles are controlled by La-ACC on a freeway-ramp network with a merging zone of $50 \mathrm{~m}$, as shown in Figure 5. On the freeway, some vehicles are randomly selected as the host vehicle and controlled by La-ACC. Using an application program interface (API), relevant traffic information from the simulator is obtained to control the host vehicles at every simulation interval of $0.2 \mathrm{~s}$. The incoming traffic on the main road and the ramp is set at approximately $1200 \mathrm{Veh} / \mathrm{h}$ and $350 \mathrm{Veh} / \mathrm{h}$, and the desired speeds of the vehicles are sampled from a truncated normal distribution (between $75 \mathrm{~km} / \mathrm{h}$ to $90 \mathrm{~km} / \mathrm{h}$ ) by the simulator.

Figure 6a illustrates the initial conditions of five vehicles, where vehicles 1 and 2 are the host vehicles, and the others are human-driven (according to the Gipps car-following model [1], which is the default control scheme in the AIMSUN simulator). The host vehicles ( 1 and 2 ) and their follower vehicles ( 3 and 4) are observed closely over a distance of $500 \mathrm{~m}$, including $250 \mathrm{~m}$ before the merging point and $200 \mathrm{~m}$ after the merging zone. With the same aggressive braking behavior of the leading vehicle (vehicle 0 ) near the merging zone, both the host vehicles are driven by the original ACC and La-ACC in independent simulation, and the corresponding results are shown in Figure 6b,c, respectively. Both ACCs have to be deactivated as the speeds fall below $20 \mathrm{~km} / \mathrm{h}$. However, the vehicles with La-ACC exhibit better speed response than that of the ACC vehicles; consequently, the followers of La-ACC vehicles also improve their speed response while approaching the merging zone.

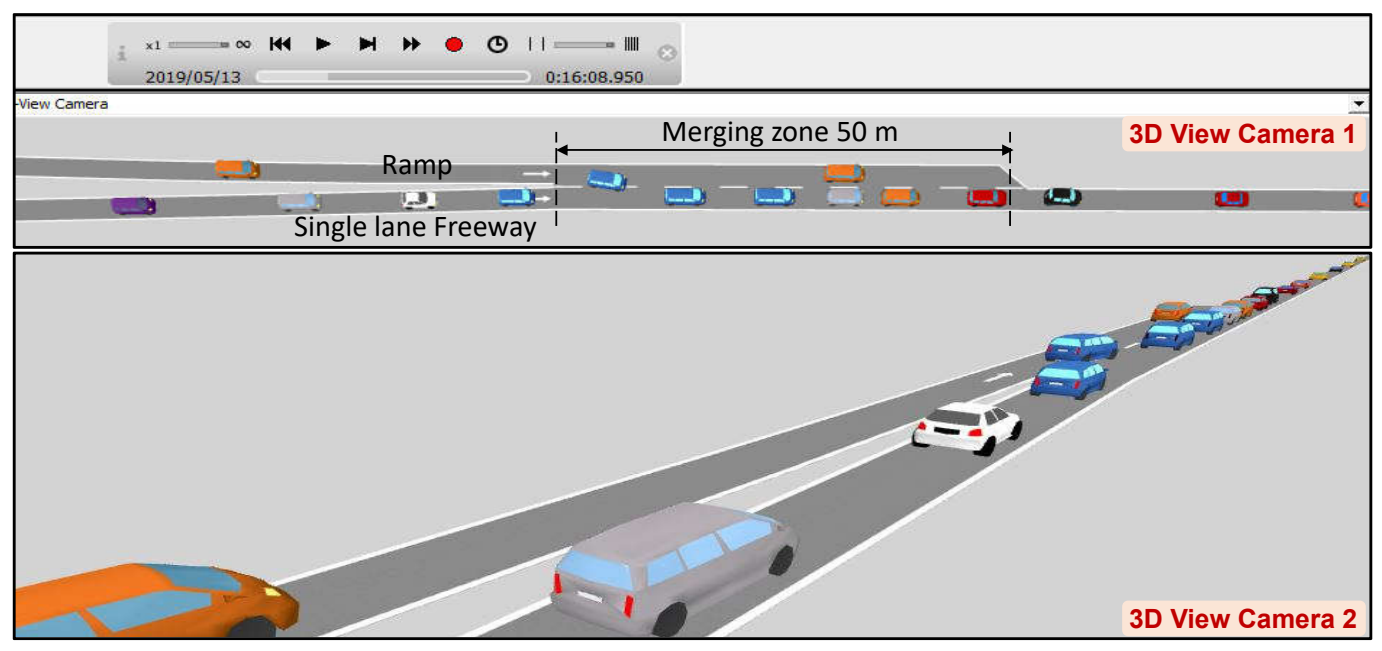

Figure 5. The simulation scenario in the AIMSUN traffic simulator viewed from two different cameras. Vehicles can merge into the freeway at any point in the merging zone of $50 \mathrm{~m}$.

For obtaining a more careful distinction between ACC and La-ACC, the average speed and fuel consumption of these five vehicles are compared in Figure $7 \mathrm{a}, \mathrm{b}$, respectively. Due to the abrupt deceleration of the leading vehicle at the merging zone, the second ACC vehicle (vehicle 2) oscillated its speed (Figure 6b); consequently, it exhibits the worst average speed and fuel consumption. However, with La-ACC (Figure 6c), both vehicles 1 and 2 show better average speed and fuel consumption; consequently, their followers, vehicles 3 and 4, also benefit from their behavior.

Finally, the impacts of a small part of La-ACC or ACC vehicles on overall traffic are investigated over a one-hour simulation with the same traffic settings to the network. In this investigation, some vehicles are randomly selected as the host vehicles, and they are driven by La-ACC or ACC in independent simulation. Since ACC vehicles are not string-stable, to avoid high penetration rates of La-ACC or ACC vehicles, the maximum number of the host vehicles is limited to two at a time on the network. Note that approximately $2.3 \%$ of vehicles are found to be controlled either by ACC or La-ACC in a one-hour simulation. 


\begin{tabular}{|llllll|}
\hline \multicolumn{7}{|c|}{} & & & \\
\hline
\end{tabular}

(a)
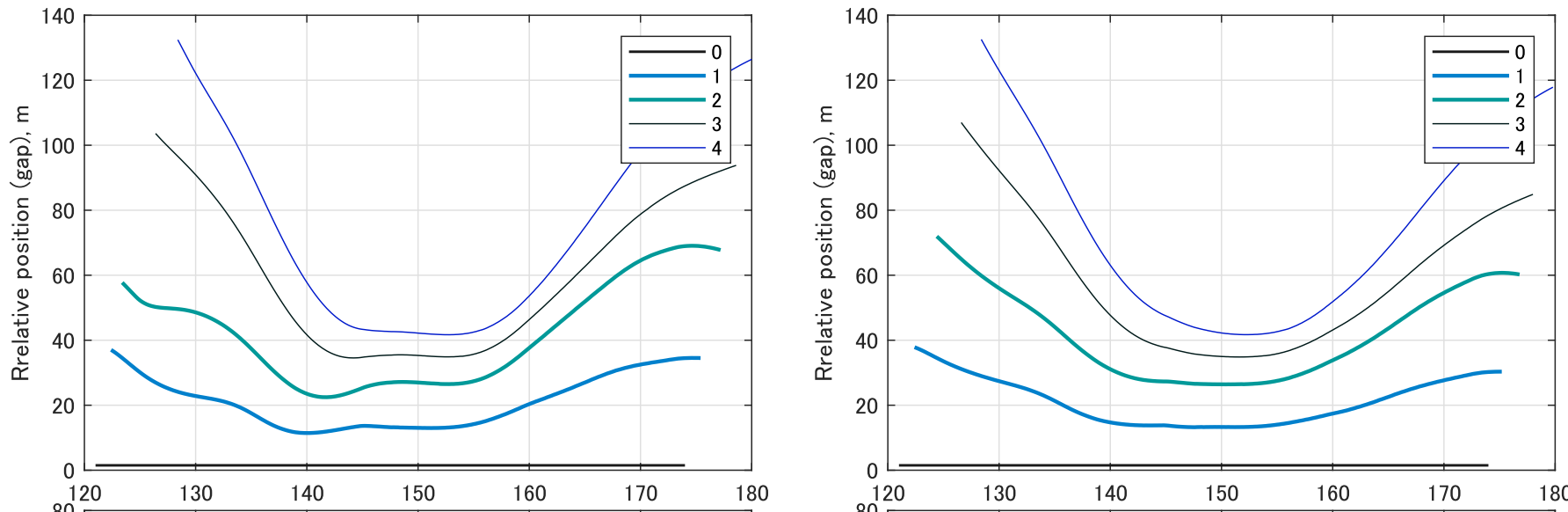

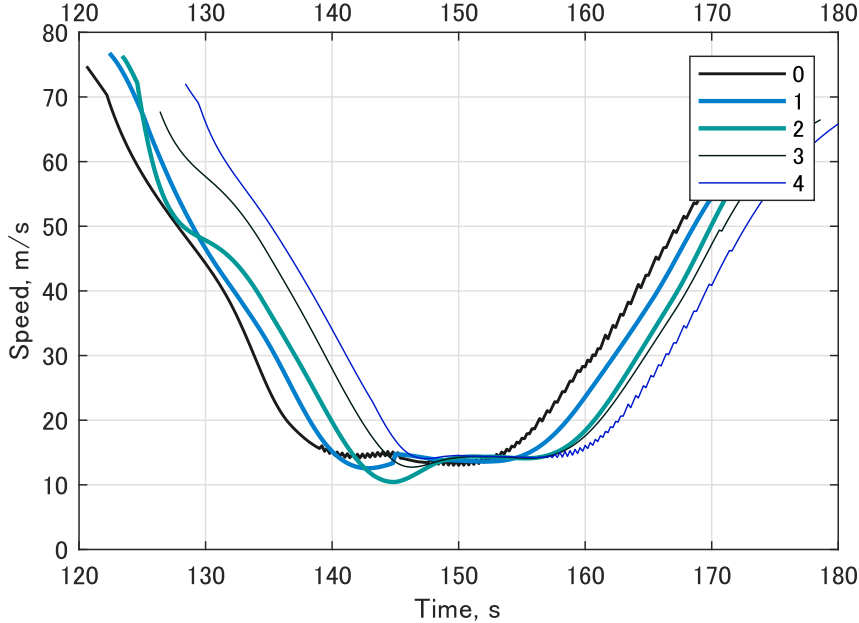

(b)

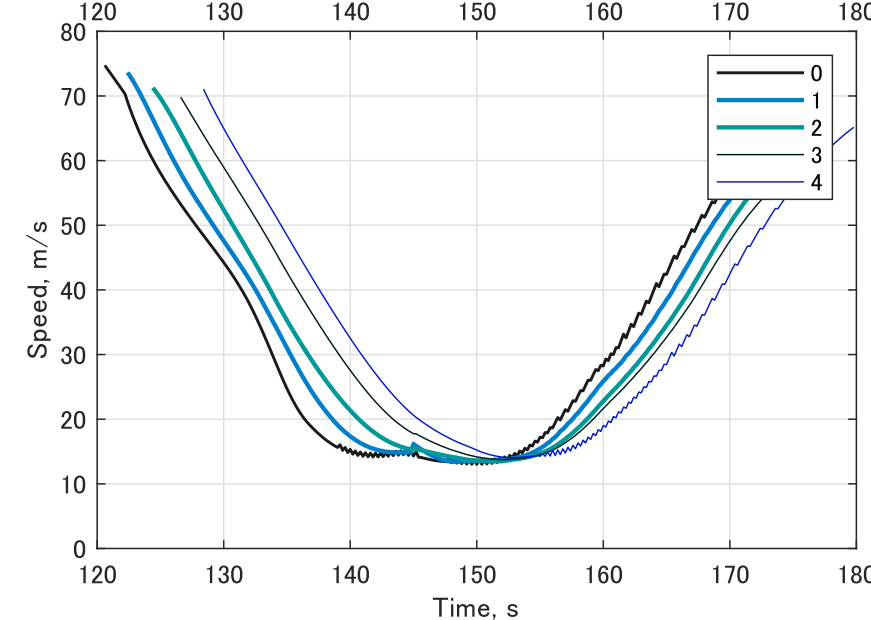

(c)

Figure 6. (a) Initial positions of the observed vehicles in the simulator. Performances of five vehicles when vehicles 1 and 2 are driven by (b) ACC and (c) La-ACC. In both cases, speed of vehicle 0 remains the same.

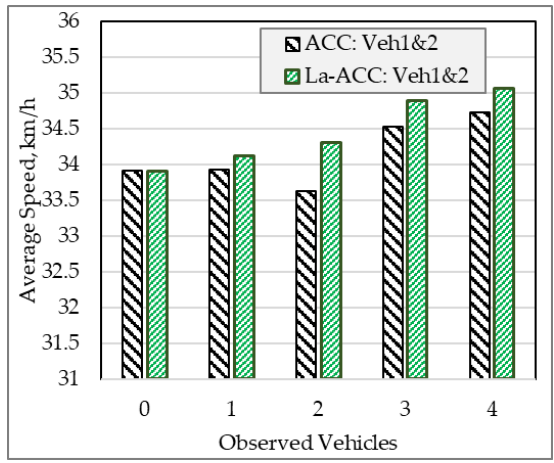

(a)

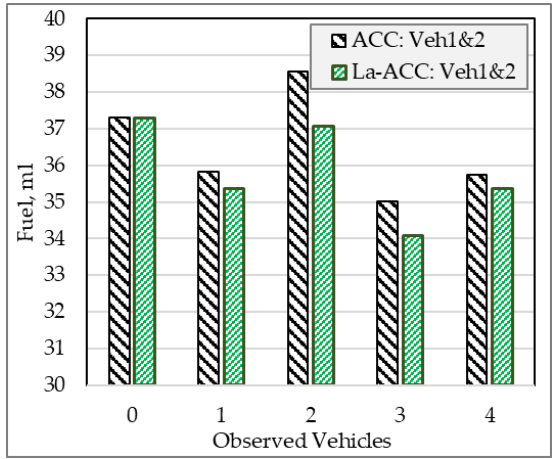

(b)

Figure 7. Individual performance when vehicles 1 and 2 are controlled by ACC or La-ACC: (a) average speed; (b) fuel consumption. 
Since traffic behavior in a network is uncertain, for a fair comparison, ten sets of one-hour simulations have been conducted independently for each ACC and La-ACC using those vehicles. Table 1 shows the average performance characteristics obtained from these simulations. From all observed features, it was found that the proposed La-ACC has positive impacts on overall traffic performance compared with ACC. The improvement margins are apparently shallow as the values are averaged for all vehicles traveling through the entire network. The use of La-ACC, instead of ACC on a small part of vehicles, improves traffic density, travel time, and fuel efficiency of the entire traffic system. Traffic delay, harmonic speed, mean queue, and other standard evaluation items are provided by the statistic generator of the AIMSUN simulator, and their computation methods can be found in the dynamic simulator manual of AIMSUN.

Table 1. The overall performances of traffic: comparison of La-ACC over ACC in mixed traffic.

\begin{tabular}{llll}
\hline Observed Features & With 2.3\% ACC Vehicles & With 2.3\% La-ACC Vehicles & Change \% \\
\hline Mean speed, km/h & 62.60 & 62.97 & $+0.59 \%$ \\
Harmonic speed, km/h & 61.04 & 61.43 & $+0.65 \%$ \\
Density, veh $/ \mathrm{km}$ & 18.61 & 18.49 & $-0.63 \%$ \\
Travel time, sec/km & 60.04 & 59.67 & $-0.61 \%$ \\
Delay, sec/km & 14.51 & 14.15 & $-2.53 \%$ \\
Stop time, sec/km & 1.99 & 1.96 & $-1.62 \%$ \\
Mean queue, veh & 0.38 & 0.37 & $-4.03 \%$ \\
Fuel efficiency, km/1 & 14.66 & 14.90 & $+1.63 \%$ \\
\hline
\end{tabular}

\section{Discussion}

This paper has presented a look-ahead scheme of ACC, called La-ACC, that is easily implementable for automated driving of a vehicle aiming at better driving performance and comfort in high-speed driving. Using the standard model of an existing ACC found on the commercial vehicles, the proposed La-ACC is evaluated. The look-ahead feature is a generic technique that introduces anticipatory behavior into the ACC or car-following models to enhance its original behavior with negligible computational complexity. Therefore, the lookahead concept is directly integrated into the car-following models. A preliminary study [34] found that the look-ahead IDM performs better than the conventional IDM in urban traffic, which flows through signalized intersections. It is expected to work well with other parametric car-following models according to our observation. Since our primary concern in this paper is to enhance the performance of ACC used in commercial cars, we do not include such investigations in this paper. However, in our future work, we would like to extensively investigate the usefulness of the look-ahead feature on different ACC or car-following models in various traffic contexts (e.g., traffic on urban roads with signalized intersections and merging scenarios). The proposed look-ahead technique should be finetuned further by using experimental driving, considering various factors associated with the natural driving environment.

The proposed La-ACC decides the control input based on prediction such as typical MPC-based driving schemes, although their prediction horizon and technique are different. Specifically, the prediction horizon of La-ACC is only a fraction of the horizon used in MPC. Instead of apprehending the predicted states of the involved vehicles in the entire horizon in MPC, the short horizon or look-ahead step in La-ACC only provides the sense of traffic trends (e.g., slowing, steady, or speeding) to help to take cautious action (i.e., not fully optimal action). La-ACC instantly decides the control input (e.g., in a few microseconds), whereas MPC needs a numerical solution of an optimization problem that usually takes a few hundreds of milliseconds.

La-ACC slightly differs from ACC because it uses the estimated acceleration of the preceding vehicle. However, the purpose of using the preceding vehicle's estimated acceleration in decision making entirely differs from CACC that aims to maintain specified gaps 
and speeds for all vehicles in the platoon. Instead of applying the part of the acceleration of the preceding vehicle directly such as in CACC, La-ACC does not use such acceleration directly in the decision making but in determining the predicted state of the preceding vehicle. This distinct feature makes La-ACC more flexible than CACC and applicable to controlling individual vehicles. In a fully connected vehicle environment, information from the multiple preceding vehicles will be available readily to better predict the behavior of the immediate preceding vehicle using some car-following models. In such a scenario, the accuracy of the look-ahead state could be better, resulting in the improved performance of La-ACC. Remarkably, a vehicle with such La-ACC can smooth traffic flows despite traffic shock waves by alleviating them in addition to improving its performance, similar to the MPC-based smart driving presented in [41]. As the main focus of this study is the existing commercial ACC in a traditional unconnected environment, such an exciting study is kept as future work.

Author Contributions: Conceptualization, methodology, and validation, M.A.S.K.; investigation, M.A.S.K.; writing—original draft preparation, M.A.S.K., K.H. and T.H.; writing-review and editing, M.A.S.K., T.H., K.Y. and J.-i.I.; supervision, T.H, K.Y. and J.-i.I.; project administration, J.-i.I. and M.A.S.K. All authors have read and agreed to the published version of the manuscript.

Funding: The authors acknowledge financial support from the Japan Society of the Promotion of Science (JSPS) Grant-in-Aid for Scientific Research (A) 18H03774 and (C) 20K04531.

Institutional Review Board Statement: Not applicable.

Informed Consent Statement: Not applicable.

Data Availability Statement: Not applicable.

Conflicts of Interest: The authors declare no conflict of interest. The funders had no role in the design of the study; in the collection, analyses, or interpretation of data; in the writing of the manuscript, or in the decision to publish the results.

\section{Appendix A. Additional Evaluation}

In addition to the evaluation of La-ACC in small speed fluctuation as shown in Figure 4, it is also evaluated against moderate speed fluctuation. Specifically, La-ACC is compared with ACC on five vehicles for the case when the first vehicle brakes at a moderate rate of $-1 \mathrm{~m} / \mathrm{s}^{2}$ (that is, a higher rate than the case shown in Figure 4) from $100 \mathrm{~km} / \mathrm{h}$ to $20 \mathrm{~km} / \mathrm{h}$, and later it accelerates typically to a speed $80 \mathrm{~km} / \mathrm{h}$ as shown in Figure A1. All vehicles have to deactivate ACC shortly when the corresponding preceding vehicle brakes as shown by the red curves. Since an ACC vehicle ignores the relative speed of its preceding vehicle and decelerates only when the gap starts reducing, they need aggressive braking to avoid a collision. Similarly, when the first vehicle speeds up, the ACC vehicles slowly accelerate at the beginning but later proceed to a higher speed to adjust the gaps with the corresponding preceding vehicles. However, such behavior eventually creates speed oscillations that ultimately force the fourth to the fifth vehicles to deactivate ACC again. In the same conditions, the La-ACC vehicles could maintain smooth flows without the necessity of deactivation of La-ACC as shown in Figure A1b. Compared with ACC vehicles, the four La-ACC vehicles are found to be more fuel efficient as they required $3.45 \%$ less fuel for the same traveling distance. These results further confirm the stable operating-range enhancement of La-ACC over ACC that is used in commercial cars. 


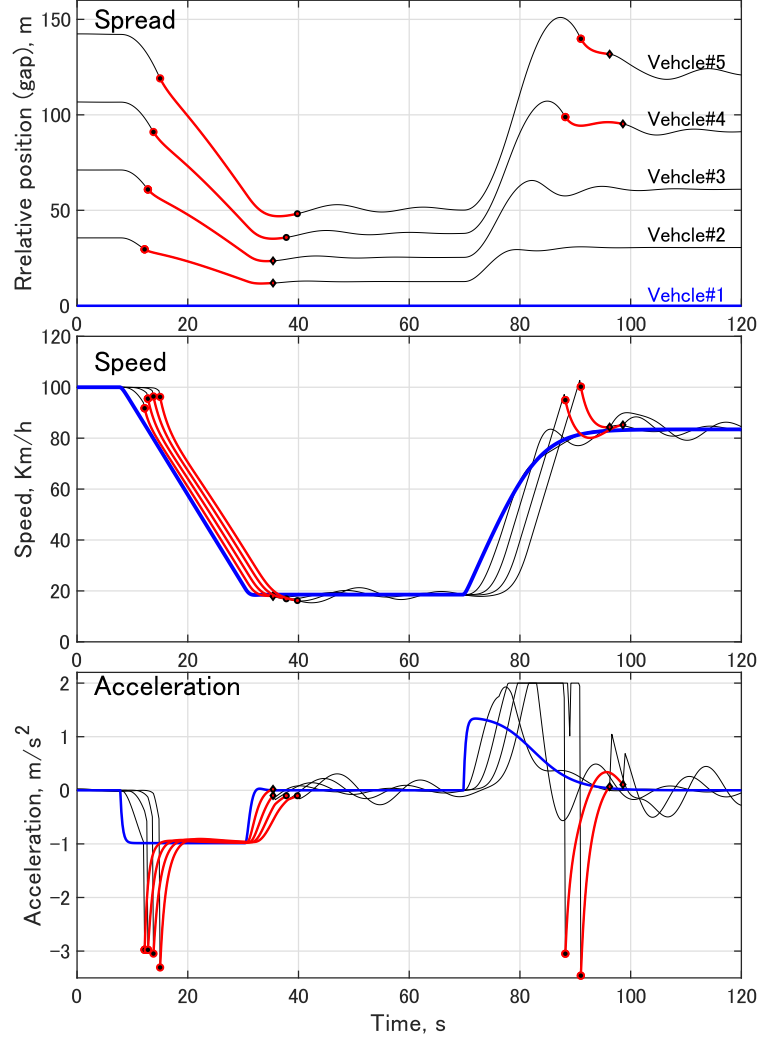

(a)
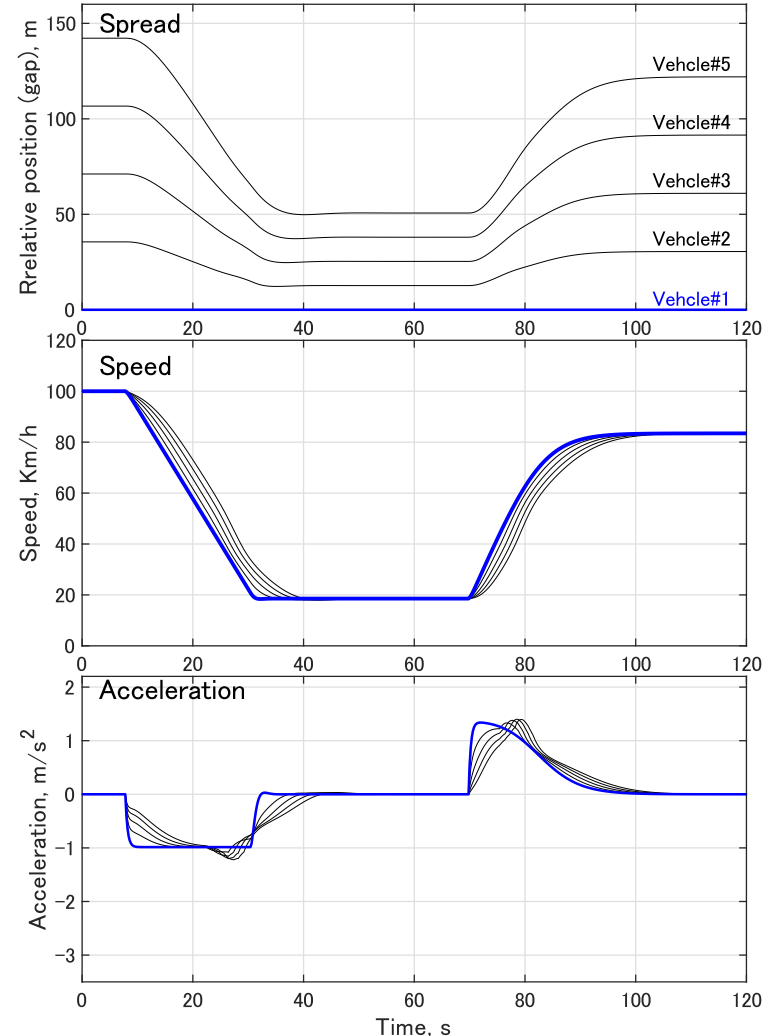

(b)

Figure A1. Flowing behavior of five vehicles when the first vehicle brakes and later accelerates at a moderate level, and the rest of vehicles are controlled by (a) ACC and (b) La-ACC. The red curves show the deactivation period as they are driven by a human driver.

\section{References}

1. Gipps, P.G. A behavioural car-following model for computer simulation. Transp. Res. Part B Methodol. 1981, 15, 105-111. [CrossRef]

2. Ge, H.; Dai, S.; Dong, L.; Xue, Y. Stabilization effect of traffic flow in an extended car-following model based on an intelligent transportation system application. Phys. Rev. E 2004, 70, 066134. [CrossRef] [PubMed]

3. Li, Z.; Li, W.; Xu, S.; Qian, Y. Stability analysis of an extended intelligent driver model and its simulations under open boundary condition. Phys. A Stat. Mech. Appl. 2015, 419, 526-536. [CrossRef]

4. Zhou, Y.; Ahn, S. Robust local and string stability for a decentralized car following control strategy for connected automated vehicles. Transp. Res. Part B Methodol. 2019, 125, 175-196. [CrossRef]

5. Marsden, G.; McDonald, M.; Brackstone, M. Towards an understanding of adaptive cruise control. Transp. Res. Part C Emerg. Technol. 2001, 9, 33-51. [CrossRef]

6. Vahidi, A.; Eskandarian, A. Research advances in intelligent collision avoidance and adaptive cruise control. IEEE Trans. Intell. Transp. Syst. 2003, 4, 143-153. [CrossRef]

7. Naranjo, J.E.; González, C.; García, R.; De Pedro, T. ACC+ Stop\&go maneuvers with throttle and brake fuzzy control. IEEE Trans. Intell. Transp. Syst. 2006, 7, 213-225.

8. Moon, S.; Moon, I.; Yi, K. Design, tuning, and evaluation of a full-range adaptive cruise control system with collision avoidance. Control Eng. Pract. 2009, 17, 442-455. [CrossRef]

9. Kesting, A.; Treiber, M.; Schönhof, M.; Helbing, D. Adaptive cruise control design for active congestion avoidance. Transp. Res. Part C Emerg. Technol. 2008, 16, 668-683. [CrossRef]

10. Li, S.; Li, K.; Rajamani, R.; Wang, J. Model predictive multi-objective vehicular adaptive cruise control. IEEE Trans. Control Syst. Technol. 2010, 19, 556-566. [CrossRef]

11. Manolis, D.; Spiliopoulou, A.; Vandorou, F.; Papageorgiou, M. Real time adaptive cruise control strategy for motorways. Transp. Res. Part C Emerg. Technol. 2020, 115, 102617. [CrossRef]

12. Davis, L. Effect of adaptive cruise control systems on traffic flow. Phys. Rev. E 2004, 69, 066110. [CrossRef]

13. Milanés, V.; Shladover, S.E.; Spring, J.; Nowakowski, C.; Kawazoe, H.; Nakamura, M. Cooperative adaptive cruise control in real traffic situations. IEEE Trans. Intell. Transp. Syst. 2013, 15, 296-305. [CrossRef] 
14. Wang, C.; Gong, S.; Zhou, A.; Li, T.; Peeta, S. Cooperative adaptive cruise control for connected autonomous vehicles by factoring communication-related constraints. Transp. Res. Part C Emerg. Technol. 2020, 113, 124-145. [CrossRef]

15. Van Arem, B.; Van Driel, C.J.; Visser, R. The impact of cooperative adaptive cruise control on traffic-flow characteristics. IEEE Trans. Intell. Transp. Syst. 2006, 7, 429-436. [CrossRef]

16. Tsugawa, S.; Kato, S.; Aoki, K. An automated truck platoon for energy saving. In Proceedings of the 2011 IEEE/RSJ International Conference on Intelligent Robots and Systems, San Francisco, CA, USA, 25-30 September 2011; pp. 4109-4114. [CrossRef]

17. Tsugawa, S.; Jeschke, S.; Shladover, S.E. A review of truck platooning projects for energy savings. IEEE Trans. Intell. Veh. 2016, 1, 68-77. [CrossRef]

18. Liang, K.Y.; Mårtensson, J.; Johansson, K.H. Heavy-duty vehicle platoon formation for fuel efficiency. IEEE Trans. Intell. Transp. Syst. 2015, 17, 1051-1061. [CrossRef]

19. Lu, C.; Dong, J.; Hu, L.; Liu, C. An Ecological Adaptive Cruise Control for Mixed Traffic and Its Stabilization Effect. IEEE Access 2019, 7, 81246-81256. [CrossRef]

20. Qin, Y.; Wang, H.; Ran, B. Stability analysis of connected and automated vehicles to reduce fuel consumption and emissions. J. Transp. Eng. Part A Syst. 2018, 144, 04018068. [CrossRef]

21. Talebpour, A.; Mahmassani, H.S. Influence of connected and autonomous vehicles on traffic flow stability and throughput. Transp. Res. Part C Emerg. Technol. 2016, 71, 143-163. [CrossRef]

22. Milanés, V.; Shladover, S.E. Modeling cooperative and autonomous adaptive cruise control dynamic responses using experimental data. Transp. Res. Part C Emerg. Technol. 2014, 48, 285-300. [CrossRef]

23. Xiao, L.; Wang, M.; Schakel, W.; Van Arem, B. Unravelling effects of cooperative adaptive cruise control deactivation on traffic flow characteristics at merging bottlenecks. Transp. Res. Part C Emerg. Technol. 2018, 96, 380-397. [CrossRef]

24. Ioannou, P.A.; Stefanovic, M. Evaluation of ACC vehicles in mixed traffic: Lane change effects and sensitivity analysis. IEEE Trans. Intell. Transp. Syst. 2005, 6, 79-89. [CrossRef]

25. Liu, H.; Wei, H.; Zuo, T.; Li, Z.; Yang, Y.J. Fine-tuning ADAS algorithm parameters for optimizing traffic safety and mobility in connected vehicle environment. Transp. Res. Part C Emerg. Technol. 2017, 76, 132-149. [CrossRef]

26. Sciarretta, A.; De Nunzio, G.; Ojeda, L.L. Optimal ecodriving control: Energy-efficient driving of road vehicles as an optimal control problem. IEEE Control Syst. Mag. 2015, 35, 71-90.

27. Nie, Z.; Farzaneh, H. Adaptive Cruise Control for Eco-Driving Based on Model Predictive Control Algorithm. Appl. Sci. 2020, 10, 5271. [CrossRef]

28. HomChaudhuri, B.; Vahidi, A.; Pisu, P. Fast model predictive control-based fuel efficient control strategy for a group of connected vehicles in urban road conditions. IEEE Trans. Control Syst. Technol. 2016, 25, 760-767. [CrossRef]

29. Zohdy, I.H.; Rakha, H.A. Intersection management via vehicle connectivity: The intersection cooperative adaptive cruise control system concept. J. Intell. Transp. Syst. 2016, 20, 17-32. [CrossRef]

30. Kamal, M.A.S.; Hayakawa, T.; Imura, J. Road-speed profile for enhanced perception of traffic conditions in a partially connected vehicle environment. IEEE Trans. Veh. Technol. 2018, 67, 6824-6837. [CrossRef]

31. Kamal, M.A.S.; Taguchi, S.; Yoshimura, T. Efficient driving on multilane roads under a connected vehicle environment. IEEE Trans. Intell. Transp. Syst. 2016, 17, 2541-2551. [CrossRef]

32. Gunter, G.; Gloudemans, D.; Stern, R.E.; McQuade, S.; Bhadani, R.; Bunting, M.; Monache, M.L.D.; Lysecky, R.; Seibold, B.; Sprinkle, J.; et al. Are Commercially Implemented Adaptive Cruise Control Systems String Stable? IEEE Trans. Intell. Transp. Syst. 2020, 22, 6992-7003. doi: :10.1109/TITS.2020.3000682. [CrossRef]

33. Treiber, M.; Hennecke, A.; Helbing, D. Congested traffic states in empirical observations and microscopic simulations. Phys. Rev. E 2000, 62, 1805-1824. [CrossRef] [PubMed]

34. Kamal, M.A.S.; Hashikura, K.; Hayakawa, T.; Ogitsu, T.; Yamada, K.; Imura, J. A Look-ahead Car Following Scheme for Efficient Driving on Urban Roads. In Proceedings of the 2020 IFAC World Congress, Berlin, Germany, 12-17 July 2020; pp. 14033-14038.

35. Kamal, M.A.S.; Hashikura, K.; Hayakawa, T.; Yamada, K.; Imura, J.i. Look-ahead Driving Schemes for Efficient Control of Automated Vehicles on Urban Roads. IEEE Trans. Veh. Technol. 2021. [CrossRef]

36. Kamal, M.A.S.; Mukai, M.; Murata, J.; Kawabe, T. Ecological vehicle control on roads with up-down slopes. IEEE Trans. Intell. Transp. Syst. 2011, 12, 783-794. [CrossRef]

37. Akçelik, R. Progress in Fuel Consumption Modelling for Urban Traffic Management; Australian Road Research Board: Singapore, 1983.

38. Schakel, W.J.; Van Arem, B.; Netten, B.D. Effects of cooperative adaptive cruise control on traffic flow stability. In Proceedings of the 13th International IEEE Conference on Intelligent Transportation Systems, Funchal,, Portugal, 19-22 September 2010; pp. 759-764.

39. Ahn, K.; Rakha, H.; Trani, A.; Van Aerde, M. Estimating vehicle fuel consumption and emissions based on instantaneous speed and acceleration levels. J. Transp. Eng. 2002, 128, 182-190. [CrossRef]

40. Barceló, J.; Casas, J. Dynamic network simulation with AIMSUN. In Simulation Approaches in Transportation Analysis; Springer: Berlin/Heidelberg, Germany, 2005; pp. 57-98.

41. Kamal, M.A.S.; Imura, J.i.; Hayakawa, T.; Ohata, A.; Aihara, K. Smart driving of a vehicle using model predictive control for improving traffic flow. IEEE Trans. Intell. Transp. Syst. 2014, 15, 878-888. [CrossRef] 